\title{
Design optimization of composite cylinders for submersible pressure hulls
}

\author{
Kechun Shen, Guang Pan \& Jun Jiang \\ School of Marine Science and Technology, Northwestern Polytechnical University, Xi'an 710072, China
}

\begin{abstract}
This study aims to optimize the design of composite cylinders for submersible pressure hulls, taking the critical buckling pressure and failure load into consideration using sub-problem approximation method in ANSYS program. The finite element analysis was verified by comparing with the test results. The ply orientation angle and the number of layers for the $i$ th ply were optimized for the composite cylinder. The effect of design variables on the optimal design was studied by sensitivity analysis. The critical buckling pressure and the load factor were analyzed and evaluated for the design variables. The optimization results showed that instead of failure load, the critical buckling pressure determined the maximum design pressure. The current results suggested that the critical buckling pressure should be taken into account along with failure load for the optimal design of composite cylinders.
\end{abstract}

KEYWORD: Composite cylinders; Optimization; Buckling; Failure

\section{INTRODUCTION}

Because of the superior mechanical characteristic and amenability to tailoring of these properties, composites have been widely used in various fields of engineering such as aerospace, automobile and wind industries [1, 2]. Reserving buoyance for underwater vehicle can be easily met by using composite materials, which have better anticorrosion and sound absorption characteristics, thus, the resistance to corrosion can be improved and the danger to be detected by sonar can be reduced [3]. Underwater vehicles subjected to external pressure are typically cylindrical and spherical. Buckling can occur for such structures, and the load capacity would be dramatically reduced. There has been considerable amount of work carried out on buckling analysis of composites structures [4-6].

Rao et al. [4] predicted the minimum buckling pressure of composite shell by two different approached i.e. the finite element model and the theoretical model. Chiachio et al. [5] presented a review on reliability of composite materials and give an overview of future direction of research on it. Bakshi et al. [6] studied first ply failure characteristics of thin composite conoidal shells using finite element approach.

As for the optimization of composites structures, a number of papers are available [7-9]. Topal et al. [7] optimized the fiber orientation of symmetrically an- gle-ply square laminated plates under uniform thermal loads and biaxial compressive. Herath et al. [8] proposed an optimization scheme to design shape adaptive laminated composite marine propellers. Fathallah et al. [9] performed the optimization of lay-up sequence using three composite materials by minimizing the buoyancy factor of submersible pressure hull.

In this study, optimization of composite cylinders under external pressure was performed. Before optimization, the work of validation of finite element analysis was conducted. The objective function was to maximize the design pressure by taking buckling pressure and failure load into account simultaneously. The ply orientation angles were taken as design variables.

\section{VALIDATION OF FINITE ELEMENT ANALYSIS}

ANSYS, commercial software for finite element analysis, was used for the buckling analysis. A total of 2225 elements, which contained 1858 8-node quadrilateral elements (SHELL 281) for the cylindrical surface and 367 10-node tetrahedral elements (SOLID187) for the right flange of the cylinder $[10,11]$, were used for the cylinder. The finite element model is shown in Figure 1. The forces speci- 
fied on the finite element were the same as those of underwater vehicles' loading condition.

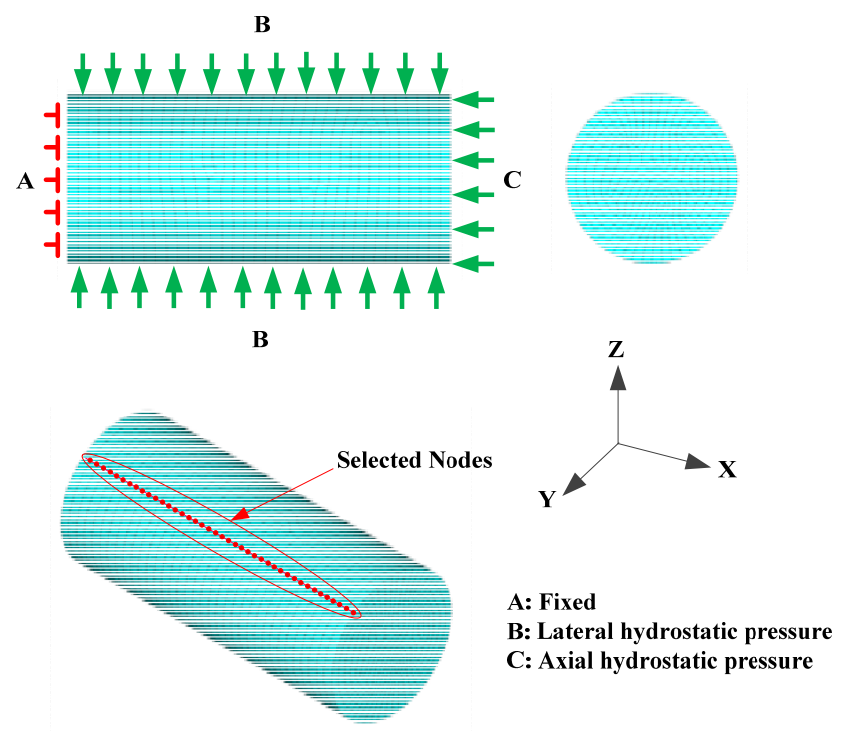

Fig.1 Finite element model

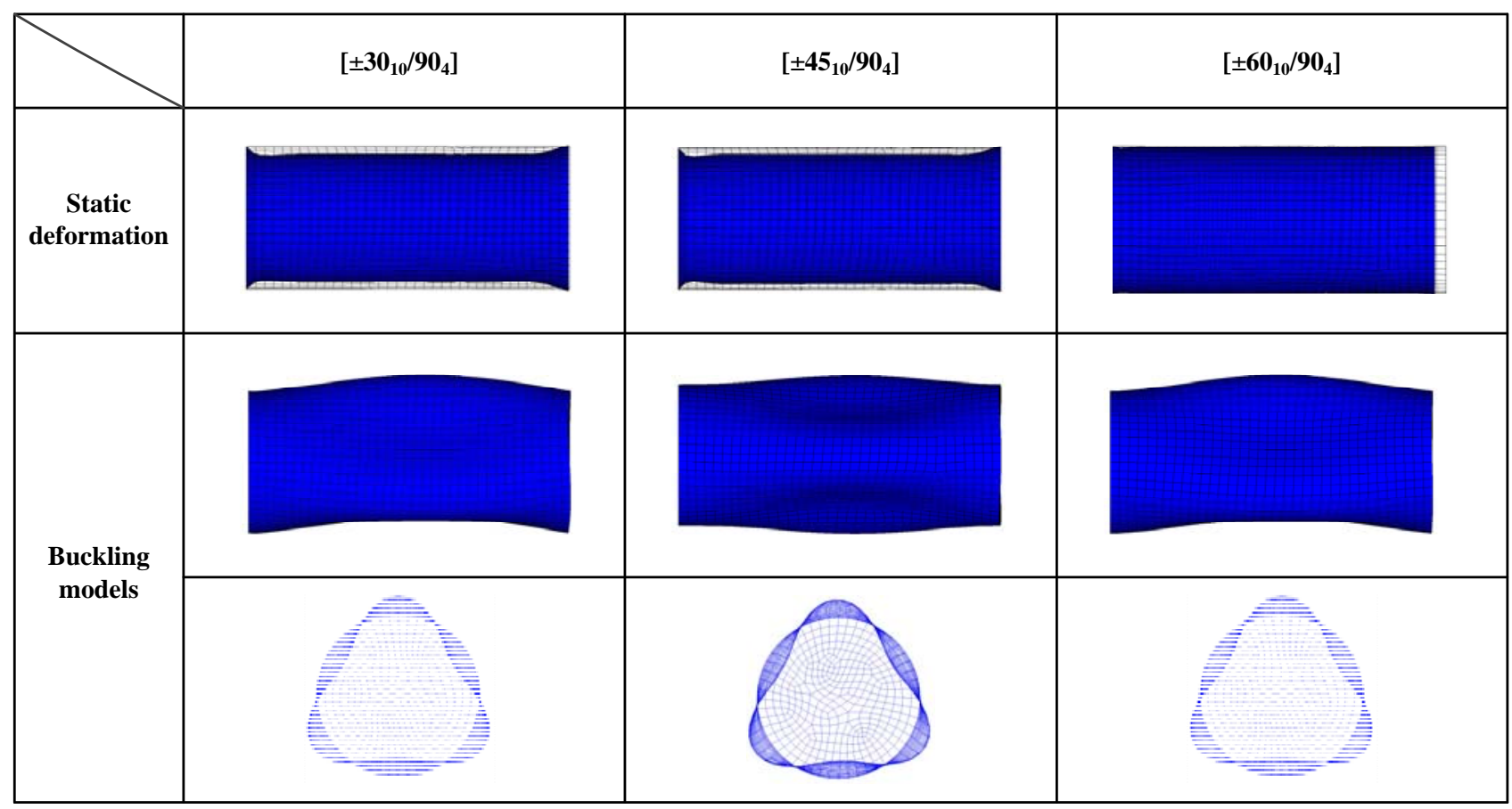

Fig. 2 Buckling model of composites cylinder

The nominal dimension of the cylinders was $300 \mathrm{~mm}$ (diameter) $\times 695 \mathrm{~mm}$ (length) and $8 \mathrm{~mm}$ thickness. Table 1 showed the material properties. The cylinders were divided into three groups according to the winding angles: $\left[ \pm 30_{10} / 90_{4}\right],\left[ \pm 45_{10} / 90_{4}\right]$ and [ $\left.\pm 60_{10} / 90_{4}\right][12]$.

Both static and stability analysis were conducted in sequence to obtain the critical buckling pressure. As shown in Figure 2, one wave exits in the axial direction and three waves in the circumferential in the buckling models of all the cylinders. As for the static deformation, in the circumferential direction, the $\left[ \pm 60_{10} / 90_{4}\right]$ cylinders are stiffer than $\left[ \pm 30_{10} / 90_{4}\right]$ and
$[ \pm 4510 / 904]$ cylinders. In the axial direction, the $\left[ \pm 60_{10} / 904\right]$ cylinders showed compression significantly and the $\left[ \pm 30_{10} / 90_{4}\right]$ cylinders performance slightly by the effect of hydrostatic pressure. The nodes (selected in Figure 1) displacement was shown in Figures 3 5. The positive deformation denotes tensile and the negative deformation denotes compression. 
Table 1 mechanical property [12]

\begin{tabular}{lll}
\hline Property & Symbol and value & Unit \\
\hline Elastic modulus & $\mathrm{E}_{11}=121, \mathrm{E}_{22}=8.6, \mathrm{E}_{33}=8.6$ & $\mathrm{GPa}$ \\
Poisson's ratio & $\mathrm{v}_{12}=0.253, \mathrm{v}_{13}=0.253, \mathrm{v}_{23}=0.421$ & - \\
Shear modulus & $\mathrm{G}_{12}=3.35, \mathrm{G}_{13}=3.35, \mathrm{G}_{23}=2.68$ & $\mathrm{GPa}$ \\
Tensile strength & $\mathrm{X}_{\mathrm{T}}=2550, \mathrm{Y}_{\mathrm{T}}=40, \mathrm{Z}_{\mathrm{T}}=40$ & $\mathrm{MPa}$ \\
Compressive strength & $\mathrm{X}_{\mathrm{C}}=2550, \mathrm{Y}_{\mathrm{C}}=40, \mathrm{Z}_{\mathrm{C}}=40$ & $\mathrm{MPa}$ \\
Shear strength & $\mathrm{S}_{12}=45, \mathrm{~S}_{13}=45, \mathrm{~S}_{23}=64$, & $\mathrm{MPa}$ \\
\hline
\end{tabular}

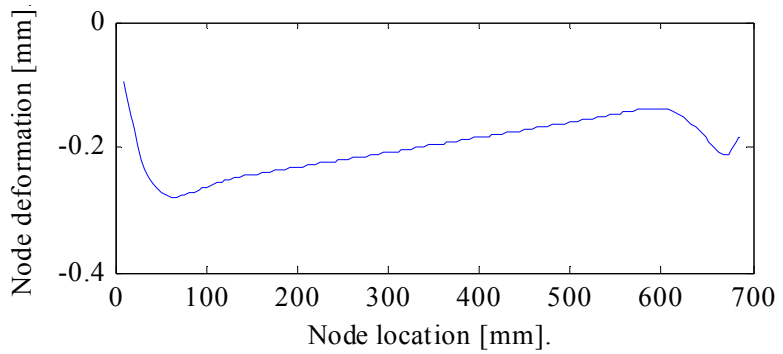

Fig. 3 Nodes deformation of $[ \pm 3010 / 904]$

Table 2 Buckling pressure [12]

\begin{tabular}{lll}
\hline ID & Stacking sequence & Experimental (Mpa) \\
\cline { 3 - 3 } & & Test \\
\hline FWT 30 & {$\left[ \pm 30_{10} / 90_{4}\right]$} & 4.13 \\
FWT 45 & {$\left[ \pm 45_{10} / 90_{4}\right]$} & 5.60 \\
FWT 60 & {$\left[ \pm 60_{10} / 90_{4}\right]$} & 7.16 \\
\hline
\end{tabular}

Table 3 Finite element buckling pressure

\begin{tabular}{lll}
\hline ID & Stacking sequence & FE buckling pressure (Mpa) \\
\cline { 3 - 3 } & & ANSYS (Error, \%) \\
\hline FWT 30 & {$\left[ \pm 30_{10} / 90_{4}\right]$} & $4.14(0.20 \%)$ \\
FWT 45 & {$\left[ \pm 45_{10} / 90_{4}\right]$} & $5.86(4.60 \%)$ \\
FWT 60 & {$\left[ \pm 60_{10} / 90_{4}\right]$} & $7.55(5.40 \%)$ \\
\hline
\end{tabular}

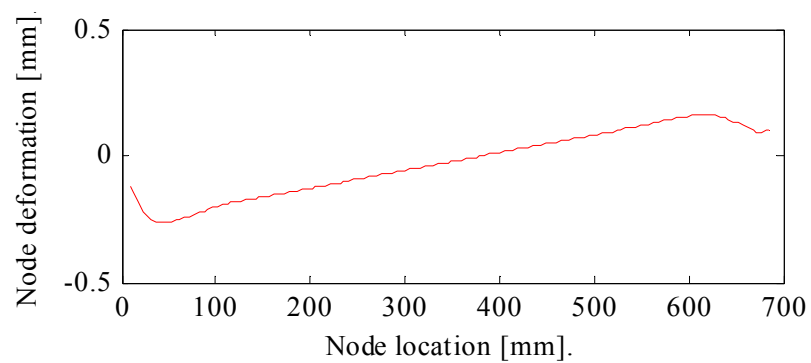

Fig. 4 Nodes deformation of $[ \pm 4510 / 904]$

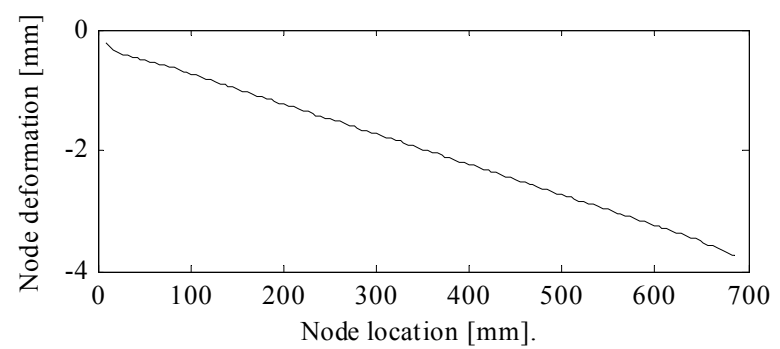

Fig. 5 Nodes deformation of $[ \pm 6010 / 904]$
Table 2 shows the experimental pressure by Moon C J [12]. Table 3 shows the finite element buckling pressure conducted by ANSYS. Comparing the finite element analysis results with experimental data, it was showed that the proposed finite element model predicts the buckling pressure with sufficient accuracy with a $5.40 \%$ deviation.

\section{OPTIMIZATION OF COMPOSITE CYLINDER}

As the proposed finite element model predicted the buckling pressure accurately enough, a study was carried out to optimize the composites cylinder taking critical buckling pressure and failure load into account. The Tsai-Wu failure criterion was used for the optimization. The objective function, design variables and design constraints were included in the optimization model, which was described as follows. The objective of the optimization was to maximize the design pressure taking critical buckling pressure and failure load into consideration. The design pressure which was regarded as the objective function was stated as follows.

Maximum design pressure $=$ Minimum $[$ Critical buckling pressure $P_{c r}$, Failure load $\left.P_{f}\right]$.

The pre-assigned design parameters was the material properties, the total number $(N=6)$ of plies, the diameter $(D=300)$ and length $(L=695)$ of the cylindrical submersible pressure hull. The ply orientation angle $\left(\theta_{1}, \theta_{2}, \theta_{3}, \theta_{4}, \theta_{5}, \theta_{6}\right)$ and the number of layers for each ply $\left(N_{1}, N_{2}, N_{3}, N_{4}, N_{5}, N_{6}\right)$ are taken as design variables.

The maximum design pressure of the composite cylindrical submersible pressure hull has two behavior constraints: one is the buckling pressure and the other is failure load.

When the hydrostatic pressure exceeds a critical value, the composite cylindrical submersible pressure hull will buckle, harming equipment and personnel. Therefore, the critical buckling pressure $\left(P_{c r}\right)$ should exceed the actual load $(P)$ to ensure the stability of the pressure hull. The constraint equation can be written as follows.

$g_{1}: \frac{P}{P_{c r}}-1 \leqslant 0$

The Tsai-Wu failure criterion was used to assess the ability of composite cylindrical hull to withstand overstressing failure. In addition, the load factor $\zeta_{k}$ was used to identify the layer failure [13]. Moreover the load factor $\zeta_{k}$ must satisfy the following condition to ensure that layer failure did not occur. While the layer failure occurred $\left(\zeta_{k}=1\right)$, the actual load that the composite cylindrical subjected to was defined as $P_{f}$. 
$g_{2}: \zeta_{k}-1 \leqslant 0 \quad k=1,2,3 \cdots$

Additionally, corresponding to the design variables, the following conditions belonged to the side constraints in this study.

$\theta_{\min } \leqslant \theta_{i} \leqslant \theta_{\max }, \quad i=1,2,3,4,5,6$

Where $\theta_{i}, \theta_{\max }$ and $\theta_{\min }$ represent the $i$ th ply orientation angle, the upper and lower bounds, respectively.

$N_{i}=4 ; \quad \sum_{i=1}^{6} N_{i}=24, \quad i=1,2,3,4,5,6$ ply.

Where $N_{i}$ represent the number of layers for the $i$ th

\section{RESULTS OF OPTIMIZATION}

Results of the optimization for the composites cylinder subjected to hydrostatic pressure were summarized in Table 4, which showed that the maximum design pressure was $10.99 \mathrm{Mpa}$ and the maximum load factor was 0.84 at layer No.9 and No.10. The optimal ply orientation angles for twenty-four layers were $\left[\begin{array}{llll}-87.44 /+77.64 & /-50.34 & /-0.94 & /-89.44 /+87.24\end{array}\right]$. The optimization results suggest that the maximum design pressure was determined by critical buckling pressure instead of failure load.

Table 4 The results of the optimal design

\begin{tabular}{cccccccccccc}
\hline Symbol & Value & Symbol & Value & Symbol & Value & Symbol & Value & Symbol & Value & Symbol & Value \\
\hline$\theta_{1}$ & $-87.4^{\mathrm{o}}$ & $N_{1}$ & 4 & $\zeta_{1}$ & 0.81 & $\zeta_{7}$ & 0.64 & $\zeta_{13}$ & 0.45 & $\zeta_{19}$ & 0.60 \\
$\theta_{2}$ & $+77.6^{\mathrm{o}}$ & $N_{2}$ & 4 & $\zeta_{2}$ & 0.73 & $\zeta_{8}$ & 0.58 & $\zeta_{14}$ & 0.45 & $\zeta_{20}$ & 0.67 \\
$\theta_{3}$ & $-50.3^{\mathrm{o}}$ & $N_{3}$ & 4 & $\zeta_{3}$ & 0.82 & $\zeta_{9}$ & 0.84 & $\zeta_{15}$ & 0.46 & $\zeta_{21}$ & 0.48 \\
$\theta_{4}$ & $-0.90^{\mathrm{o}}$ & $N_{4}$ & 4 & $\zeta_{4}$ & 0.72 & $\zeta_{10}$ & 0.84 & $\zeta_{16}$ & 0.46 & $\zeta_{22}$ & 0.53 \\
$\theta_{5}$ & $-89.4^{\mathrm{o}}$ & $N_{5}$ & 4 & $\zeta_{5}$ & 0.65 & $\zeta_{11}$ & 0.83 & $\zeta_{17}$ & 0.63 & $\zeta_{23}$ & 0.59 \\
$\theta_{6}$ & $+87.2^{\mathrm{o}}$ & $N_{6}$ & 4 & $\zeta_{6}$ & 0.65 & $\zeta_{12}$ & 0.83 & $\zeta_{18}$ & 0.71 & $\zeta_{24}$ & 0.65 \\
$P_{c r}$ & $10.99 \mathrm{Mpa}$ & & & & & & & & & & \\
\hline
\end{tabular}

Sensitivity analysis was conducted to elucidate the effects of design variables on the objective of optimization. Figures 6-12 presented the results.

Figures 6-11 showed the effect of ply orientation $\theta_{i}$ upon the critical buckling pressure. In case of $\theta_{1}$, the critical buckling pressure obtained its maximum value at the angle of -85 degree and got the minimum value at the angle of -10 degree. In case of $\theta_{2}$, the critical buckling pressure got the maximum value at the angle of 80 degree. Within the domain of function, the critical buckling pressure presented decreasing trends in the first half of the domain, and then rose sharply in the second half. As for the $\theta_{3}$, three extreme points existed within the domain of function. As shown in Figure 9, the $\theta_{4}$ vs. $P_{c r}$ cure was bell-shaped which looks like the Gaussian distribution and centered on 10 . For $\theta_{5}$, the maximum critical buckling pressure occurred at the angle of -90 degree. As for $\theta_{6}$, which was almost the same as that of $\theta_{5}$.

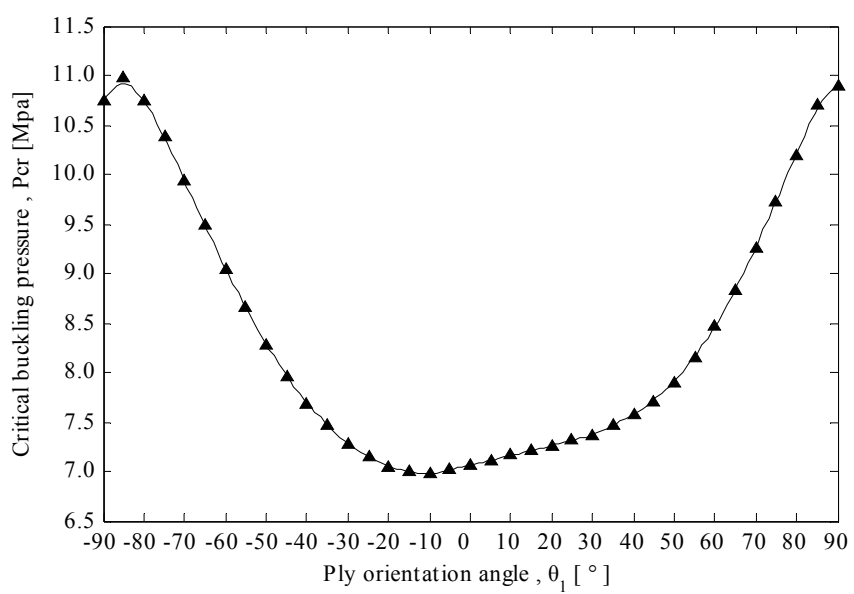

Fig. $6 \theta_{1}$ upon the $P_{c r}$

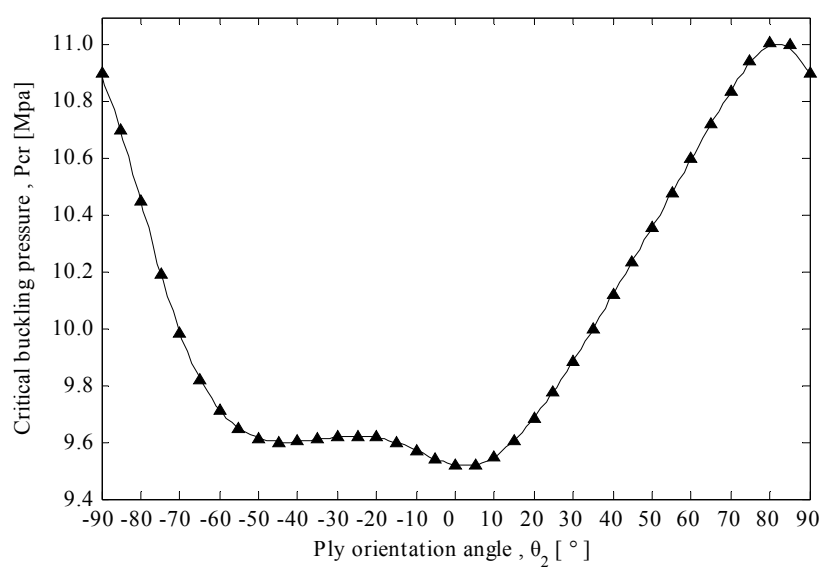

Fig. $7 \theta_{2}$ upon the $P_{c r}$ 


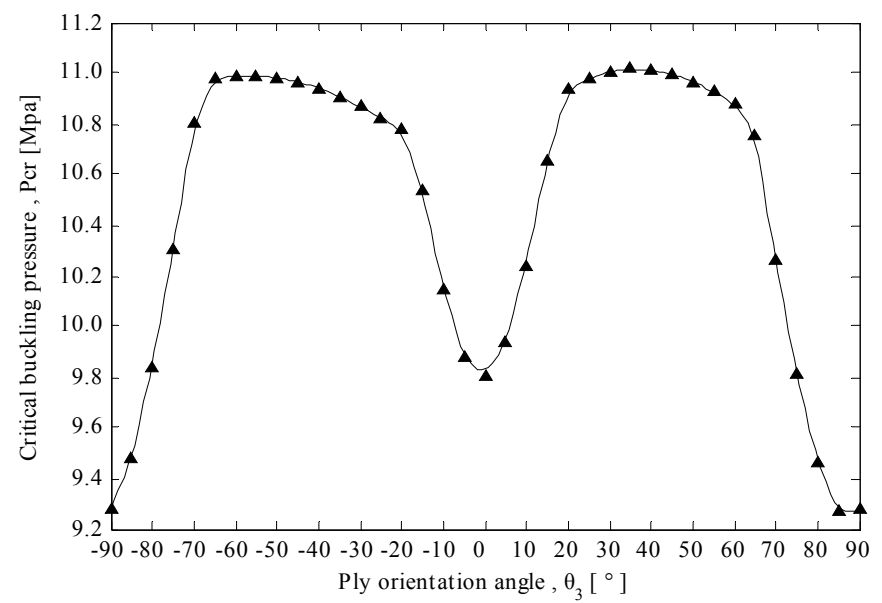

Fig. $8 \theta_{3}$ upon the $P_{c r}$

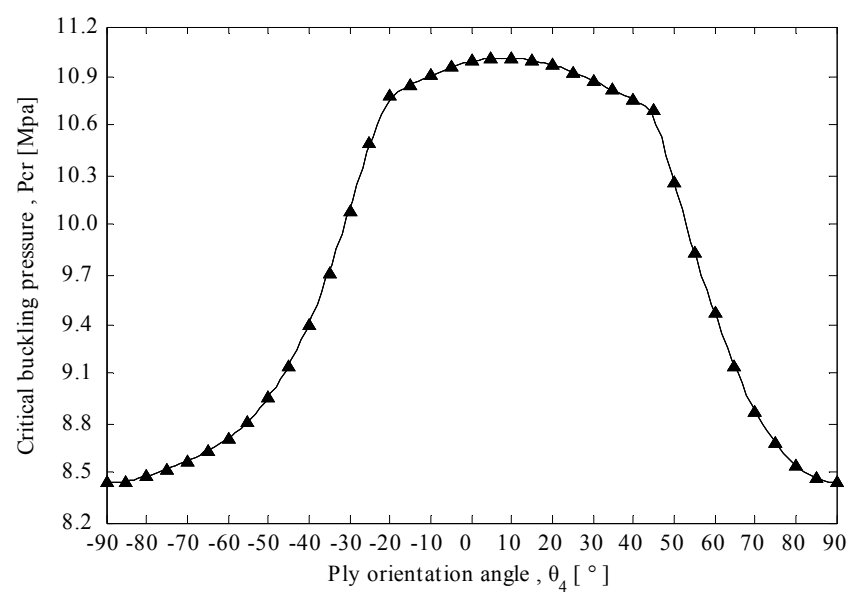

Fig. $9 \theta_{4}$ upon the $P_{c r}$

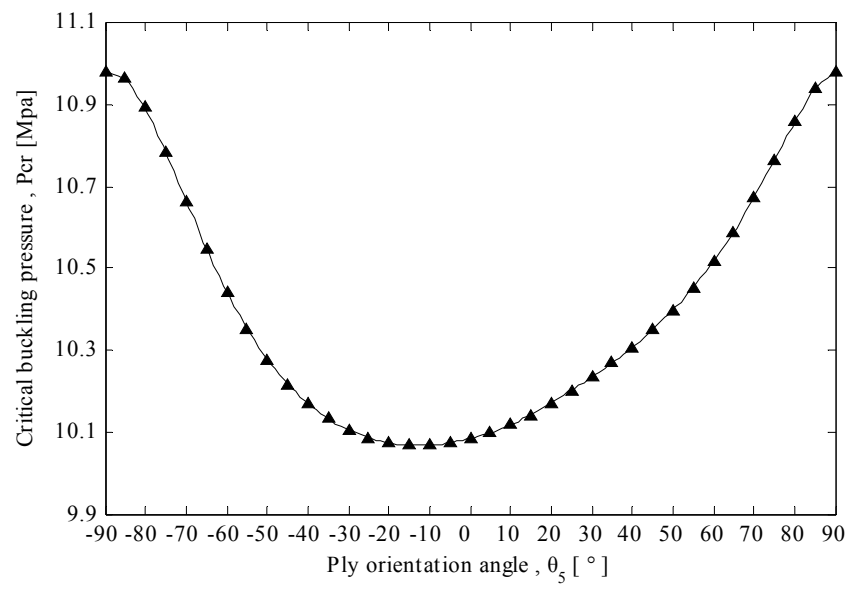

Fig. $10 \theta_{5}$ upon the $P_{c r}$

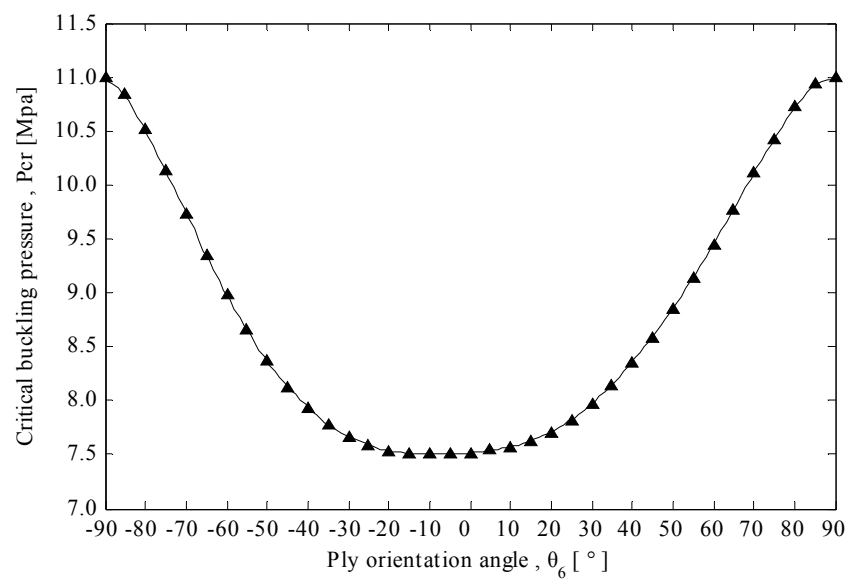

Fig. $11 \theta_{6}$ upon the $P_{c r}$

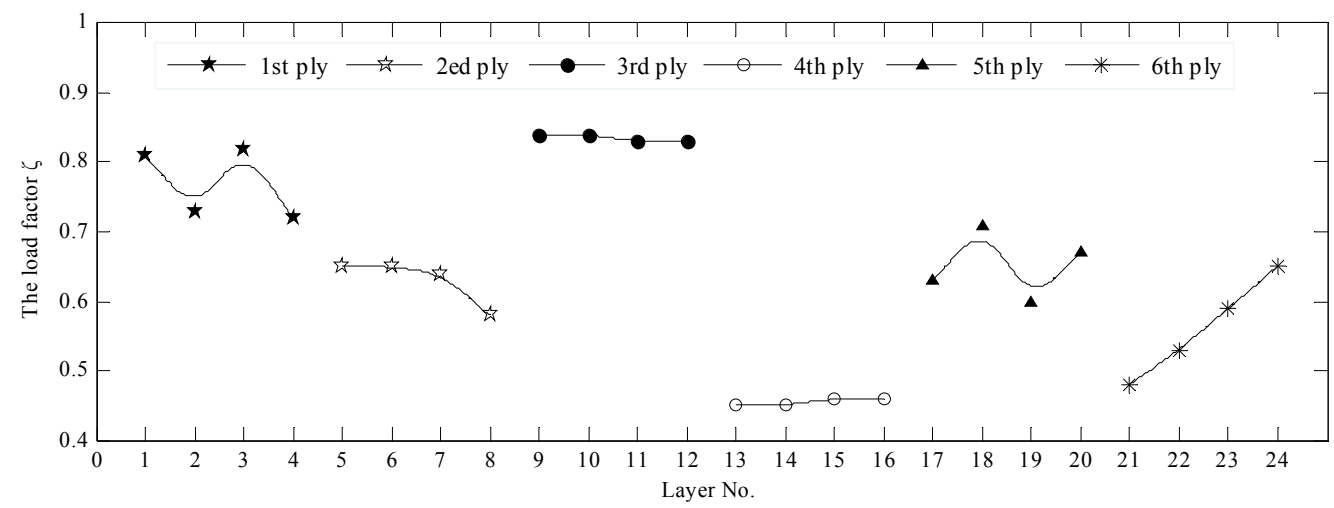

Fig. 12 The load factor $\zeta$ of each layer

Figure 12 showed the value of load factor $\zeta$ of each layer. For the first ply, the orientation angle was $-87.4^{\circ}$. In case of the second ply, the orientation angle was $77.6^{\circ}$. For the third ply, the layer failure occurred was most serious as the load factor $\zeta$ got the maximum value which was basically a constant around 0.83 . For the fourth ply, the load factor $\zeta$ was around 0.45 and the layer failure occurred was slight. The changing trends of the load factor $\zeta$ in the fifth ply were similar as that in the first ply. As for the sixth ply, the layer failure got worse layer by layer.

\section{CONCLUSION}

This paper addressed the optimum design of composite cylinders under external hydrostatic pressure. Both critical buckling pressure and failure load were taken into consideration. Maximum design pressure for composite cylinders under external hydrostatic pressure was considered as the objective function. The ply orientation angles $\theta_{i}$ are taken as design variables. A sensitivity analysis was performed to 
study the effect of design variables on the optimal design. The results obtained emphasize a vital fact that considering the critical buckling pressure and failure load, the maximum design pressure was determined by the critical buckling pressure instead of the failure load.

\section{ACKNOWLEDGMENTS}

This work was supported by the National Natural Science Foundation of China (Grant No. 51279165) and Prospective Project of Northwestern Polytechnical University (Grant No. 3102015BJ012). Corresponding author: Pan Guang (email:panguang601@163.com).

\section{REFERENCES}

[1] Omkar SN, Senthilnath J, Khandelwal R, Naik GN, Gopalakrishnan S. Artifial Bee Colony (ABC) for multi-objective design optimization of composite structures. Appl Soft Comput 2011; 11:489-499.

[2] Shen GL, Hu GK, Mechanics of composite materials. Tsinghua University Publishing House: Beijing, 2006.

[3] Ross CTF. A conceptual design of an underwater vehicle. Ocean Eng 2006; 33:2087-2104.

[4] Rao YKSS, Mohan RK, Kiran BV. Composite pressure vessels. Int J Res Eng Technol 2012; 1(4):597-618

[5] Chiachio M, Chiachio J, Rus G. Reliability in compositesA selective review and survey of current development. Compos Part B: Eng 2012; 43:602-913.

[6] Bakshi K, Chakravorty D. First ply failure study of thin composite conoidal shells subjected to uniformly distributed load. Thin-walled Struct 2014; 76:1-7.

[7] Topal U, Uzman U. Multiobjective optimization of angleply laminated plates for maximum buckling load. Finite Elem Anal Des 2010; 46:273-279.

[8] Herath MT, Natarajan S, Prusty BG, St John N. Smoothed finite element and genetic algorithm based optimization for shaped adaptive composite marine propellers. Compos Struct 2014; 109:189-197.

[9] Fathallah E, Qi H, Tong LL, Helal M. Design optimization of lay-up and composite material system to achieve minimum buoyancy factor for composite elliptical submersible pressure hull. Compos Struct 2015; 121:16-26.

[10] A. Inc. "ANSYS Theory Reference Release 14.5." October 2012

[11] Madenci E, Guven I. The Finite Element Method and Applications in Engineering Using ANSYS, http://link.springer.com/book/10.1007/978-1-4899-7550-8

[12] Moon CJ, Kim IH, Choi BH, et al. Buckling of filamentwound composite cylinders subjected to hydrostatic pressure for underwater vehicle applications. Compos Struct 2010; 92: 2241-2251

[13] Tsai SW, Hahn HT. Introduction to Composite Materials. Technomic Publishing Co, Inc. Lancashire, England. 1980.277-327. 